\title{
Dinámicas No-Lineales y No-Suaves en Procesos Estrés-Enfermedad
}

\section{Nonlinear and Nonsmooth Dynamics in Stress-Sickness Processes}

\author{
Jorge Armando Amador Moncada ${ }^{a^{*}}$ \\ Héctor Andrés Granada Díaz ${ }^{b}$ \\ Johan Manuel Redondo Ortegón ${ }^{c}$ \\ Gerard Olivar Tost ${ }^{\mathrm{d}}$
}

Recepción: 26 de Septiembre de 2016

Aceptación: 30 de Diciembre de 2016

\begin{abstract}
Resumen
De acuerdo a referencias, existe una fuerte influencia del estrés con el desarrollo de enfermedades. En este trabajo se consideraron los atributos que hacen posible esa influencia para la elaboración de un modelo matemático que permite el planteamiento de escenarios que cambian con la frecuencia con la que un individuo implementa soluciones temporales para atender el estrés (pseudosoluciones) o cambia la frecuencia con la que recibe el tratamiento médico especializado para atender la enfermedad. Los resultados son concluyentes de la dinámica del comportamiento del sistema, permitiendo la configuración de escenarios en los que es posible que el paciente: 1) sane completamente, 2) alcance niveles estables de estrésenfermedad que puede sobrellevar sin riesgos pero con la implementación de diferentes pseudosoluciones y tratamientos médicos, o 3) ponga en riesgo su vida.
\end{abstract}

Palabras clave: Estrés, dinámica de sistemas, sistemas dinámicos no suaves, método convexo de Filippov.

\begin{abstract}
According to literature, there is a strong influence of stress with the emergence of diseases. In this paper the attributes that make possible this influence in order to develop a mathematical model that allows the approach of scenarios that change with the frequency an individual implements temporary solutions to deal with stress (pseudosolutions), or change the frequency with which it receives the specialized medical treatment to treat the disease were considered. Results are conclusive of the dynamic behavior of the system, allowing the configuration of different scenarios in which the individual: 1) is completely healed, 2) reaches stable levels of stress-sickness which can bear safely but with the implementation of different pseudosolutions and medical treatments, or 3) jeopardizes its life.
\end{abstract}

Key words: Stress, system dynamics, non-smooth dynamical systems, Filippov convex method.

\footnotetext{
${ }^{a}$ Departamento de Ingeniería Eléctrica, Electrónica y Computación, Universidad Nacional, Manizales, Colombia.

*Autor de correspondencia: jaamadorm@unal.edu.co

${ }^{b}$ Departamento de Matemáticas y Estadística, Universidad del Tolima, Manizales, Colombia.

${ }^{\mathrm{c}}$ Departamento de Matemáticas, Universidad Serio Arboleda, Bogotá, Colombia.

${ }^{\mathrm{d}}$ Departamento de Matemáticas y Estadística, Universidad Nacional, Manizales, Colombia.
} 


\section{Introducción}

Las primeras ideas sobre la influencia que tiene el estrés en el desarrollo de enfermedades se remonta a escritos médicos de los asirios, griegos y romanos. Esas contribuciones se documentaron en informes de casos aislados o pequeños grupos de pacientes. Pese a sus persuasivos resultados, modernamente se requerían evidencias más contundentes [1]. Es así como en el siglo $X X$ se logró identificar una relación entre eventos estresantes como el calor, el frío y el hambre, con respuestas psicológicas a estos eventos.

Un progreso importante del siglo XX, fue la diferenciación de los eventos estresantes en generales y específicos. Hans Selye insistió en la influencia de estresantes generales importantes en muchas enfermedades. Algunos ejemplos son calor, frio y hambre [2]. Walter B. Canon, cuyo trabajo sobre la relación entre las emociones y respuesta psicológica precediá a los estudios de Selye, documentó el efecto de estresantes específicos sobre los sistemas corporales. Utilizó como ejemplos el ladrido de un perro, que interrumpía la digestión del gato durante una hora [3]. Recientemente, se documentó la relación "estresante específico" y "respuesta psicológica" en estudios realizados en ratas [4]. James Henry enumeró patrones de respuesta al estrés en función de las diferentes percepciones de los estresantes [5].

Ransom Arthur diseñó un modelo de estrés y afrontamiento, denominado "modelo de estrés y afrontamiento", en el cual se explica en una secuencia de seis pasos, como el estrés puede llegar a generar enfermedades. Su modelo (no matemático) es un proceso basado en estadísticas epidemiológicas que explican los diferentes estados por los que pasa un individuo con síntomas de estrés [6].

Típicamente, el estrés es una repuesta somática a un estímulo psicológico, generador de enfermedad. Los síntomas del estrés son el reflejo de una situación problema subyacente que resulta difícil de atender, bien sea porque no se conoce bien, porque toma tiempo atenderla, porque resulta costoso afrontarlo o porque es muy engorroso hacerlo, razón por la cual se hace frecuente el tratamiento de los síntomas del estrés y se deja de lado la atención del problema fundamental generador de estrés.

Un ejemplo muy representado con Dinamica de Sistemas $[8,9,10]$ acerca del estrés, es el provo- cado por el exceso de trabajo en un individuo. La primera solución que viene a la mano es la solución sintomática, sin embargo el estrés reincide y el individuo debe incrementar gradualmente las cantidades o tiempos de esa solución sintomática, cayendo, sin percibirlo, en un círculo vicioso de adicción.

En nuestro trabajo, hemos construido un modelo matemático determinístico no-lineal del modelo de Ransom Arthur, teniendo como punto de partida el modelo reportado en [11], de la forma $\dot{x}=f\left(x_{1}, x_{2}, \mu\right)$, donde $x_{1}$ representa el nivel de estrés, $x_{2}$ el nivel de enfermedad, y $\mu$ los parámetros del sistema, utilizando la metodología de modelamiento de la dinámica de sistemas. Después, hemos particionado el plano de estados, configurando un sistema de Filippov [12] o sistema discontinuo por tramos, de modo que, el modelo pueda ser utilizado para el estudio de la evolución de enfermedades que derivan de la respuesta psicológica frente a eventos estresantes. Los resultados obtenidos en el sistema de Filippov sugieren multiplicidad de estados estacionarios donde intervienen fenómenos como deslizamientos y la existencia de pseudo-equilibrios.

Las aplicaciones de los sistemas de Filippov son muy diversas. En ingeniería se ha utilizado para modelar motores eléctricos y convertidores de potencia [13]. También se ha utilizado para modelar sistemas donde a través de cambios abruptos en la dieta o en el hábitat se controla una determinada población [14] o sistemas que representan la explotación de algún recurso natural cuando su extracción se prohíbe al alcanzarse el máximo de explotación permitido [15].

Este artículo está organizado de la siguiente manera: En la Sección (2) se presentan las hipótesis dinámicas tenidas en cuenta para la elaboración del modelo, se distinguen las variables de estado, las razones de cambio, las variables auxiliares y los parámetros con los cuales se elaboran las ecuaciones, que conducen, finalmente, a la primera versión del modelo matemático de nuestro problema. En la Sección (3) se discute sobre la representación de los campos vectoriales en las regiones de conmutación, presentando el método convexo de Filippov [12] y la forma en que este método será utilizado para establecer la dinámica en los puntos de corte de las regiones de conmutación. En la Sección (4) construiremos el sistema discontinuo a tramos que es nuestra versión definitiva del modelo matemático del problema. En 
la Sección (5) se discuten los resultados de algunas simulaciones representativas realizadas y de su aplicación en la relación estrés-enfermedad. Finalmente, en la Sección (6) se presentan las conclusiones del trabajo y en la Sección (7) las referencias.

\section{Modelo matemático preliminar}

Para la construcción del modelo matemático, tuvimos en cuenta un conjunto de atributos que nos permitieran acercarnos al problema estrés-enfermedad. Los atributos considerados son los siguientes:

1. Estresor: es un concepto asociado a todas las cargas o exigencias externas que desencadenan estrés ${ }^{1}$. Pueden ser biogénicos cuando provienen del medio ambiente o de procesos internos del organismo, o psicosociales cuando las reacciones del cuerpo provienen de la interpretación cognitiva o de los significados sociales que damos a las situaciones en las que nos involucramos ${ }^{2}$. El estresor está condicionado por la intensidad, la duración y la frecuencia ${ }^{3}$. En este trabajo haremos referencia a los estresores psicosociales.

2. Defensa psicológica: es una respuesta psicológica que experimentan los individuos frente al estresor. Un sujeto puede utilizar uno o más mecanismos psicológicos de defensa. Algunos como represión y negación, difractan señales que en estados de indefensión estimularían diversas respuestas psicofisiológicas.

3. Psicosomatización: es el proceso mediante el cual transformamos involuntariamente estados psicológicos en estados corporales provocando daños orgánicos reales. Entre los psicosomatismos tenemos la tensión muscular y las cefaleas.

4. Pseudosolución: es el tipo de solución asociado a la atención de los síntomas del estrés. Se le

\footnotetext{
${ }^{1}$ Harrison. Principios de Medicina Interna, Anthony Fauci, 17a ed. 2008, McGraw-Hill.

${ }^{2}$ Dr. Jorge A. Grau Abalo. Estrés, personalidad, salud y enfermedad. Material docente para la Maestría en Medicina Bioenergética y Natural en la Atención Primaria de Salud, Instituto Superior de Ciencias Médicas de la Habana, 2004.

Dra. Marta Martén Carbonell. Estrés, personalidad, salud y enfermedad. Material docente para la Maestría en Medicina Bioenergética y Natural en la Atención Primaria de Salud, Instituto Superior de Ciencias Médicas de la Habana, 2004.

${ }^{3}$ L. De Rivera, Los síndromes de estrés, Madrid, 2010
}

denomina pseudo o falsa, porque es una solución que atiende los síntomas del problema y no el problema en sí mismo.

5. Tratamiento médico: es la solución fundamental para atender la enfermedad provocada por el estrés, se requiere de un tratamiento médico, que tradicionalmente no busca causas subyacentes a los síntomas, escondiendo la relación existente con el estrés.

6. Enfermedad: usualmente, la enfermedad ha sido definida como ausencia de salud, entendiéndose esto como ausencia de bienestar físico, psíquico y social. En este documento diremos que la enfermedad es un estado del sistema de un ser humano, caracterizado por valores anormales de las funciones de estado tanto cuantitativas y cualitativas, que afectan a todo el sistema del ser humano ${ }^{4}$.

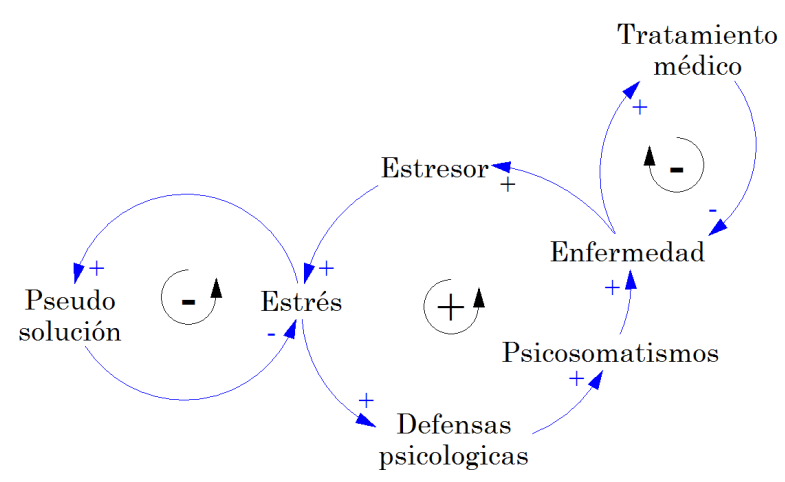

(a)

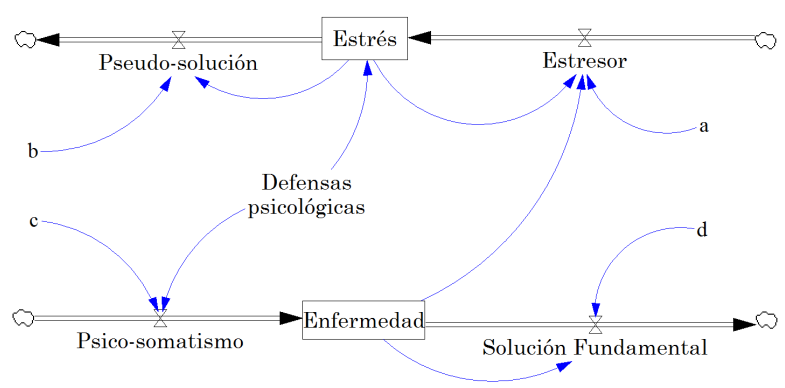

(b)

Figura 1. Diagrama causal, niveles y flujos.

Siguiendo la metodología de modelamiento de la dinámica de sistemas [8, 9], en la Figura (1(a))

\footnotetext{
${ }^{4}$ Adolfo Peña, Ofelia Paco, 2003. El concepto general de enfermedad. Revisión, crítica y propuesta Tercera parte: un modelo teórico de enfermedad. Anales de la Facultad de Medicina Universidad Nacional Mayor de San Marcos. ISSN 1025-5583, Vol. 64, No 1, Págs. 55 - 62.
} 
se ha construido un diagrama de causas y efectos con los atributos explicados, en el cual se muestra que cuando un individuo entra en estado de estrés, inconscientemente provoca el aumento de sus defensas psicológicas, produciendo psicosomatismos, que terminan desencadenando el estado de enfermedad del individuo. Al aparecer con la enfermedad, el individuo posee un nuevo elemento estresor que proviene de la misma enfermedad, de modo que seguirá alimentando su estado de estrés. De esta manera se configura el bucle de realimentación positivo presentado en el centro de la Figura (1(a)). El bucle de realimentación negativo de la izquierda presenta el estrés como el síntoma de un problema, que se resuelve con pseudosoluciones o soluciones sintomáticas. Finalmente, el bucle de realimentación negativo de la derecha emerge al relacionar la enfermedad con un tratamiento médico orientado a sacar al individuo del estado de enfermedad, de modo que el aumento de la enfermedad aumenta el tratamiento médico y éste, a su vez, disminuye la enfermedad.

Cada relación causal en la Figura (1(a)) constituye una hipótesis dinámica del sistema y, por lo tanto, una hipótesis para la configuración del modelo matemático buscado.

Continuando con la metodología de la dinámica de sistemas, en la Figura (1(b)) se presenta lo que se conoce con el nombre de diagrama de niveles y flujos, donde se definen las variables de estado, las razones de cambio, las variables auxiliares y los parámetros del sistema en coherencia con el diagrama causal de la Figura (1(a)). Se nota en la Figura (1(b)), que las variables de estado que serán consideradas en este documento son el estrés y la enfermedad.

A partir del diagrama de niveles y flujos, en concordancia con la dinámica de sistemas, presentaremos a continuación las ecuaciones del modelo con las que se elaboró el modelo matemático buscado. Las ecuaciones de nivel son:

$$
\begin{aligned}
& \frac{d}{d t} \text { Estrés }=\text { Estresor }- \text { Pseudosolución, } \\
& \frac{d}{d t} \text { Enfermedad }=\text { Psicosomatismos }- \text { Tratamiento médico. }
\end{aligned}
$$

Las ecuaciones de flujo son:

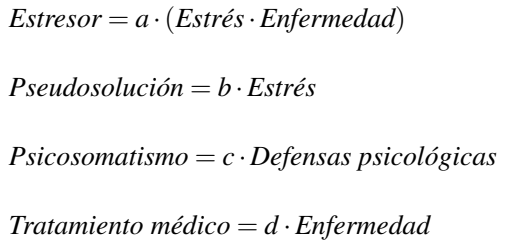

Cada uno de los parámetros utilizados se ha considerado como estrictamente mayor que cero. $a$ es la tasa de incremento del estrés a partir de la enferme$\mathrm{dad} ; b$ representa la frecuencia con la que se recurre a pseudosoluciones para aliviar el estrés; $c$ indica la frecuencia en la que se realiza la psicosomatización; y $d$ es la frecuencia en la que se sigue un tratamiento médico a partir de la enfermedad.

La ecuación de la variable auxiliar defensas psicológicas, es una función que depende del estrés

\section{Defensas psicológicas $=f($ Estrés $)$}

Por simplicidad, definimos la función $f$ considerando que la relación entre el estrés y las defensas psicológicas es directamente proporcional, con constante de proporcionalidad $k$, lo cual define una relación lineal entre las variables [6], de la forma

$$
\text { Defensas psicológicas }=k \cdot \text { Estrés }
$$

Dado que no es trivial definir en la practica el valor de la constante de proporcionalidad $k$ y por simplicidad, se tomará para el resto del documento $k=1$.

Reemplazando la ecuación auxiliar (3) en las ecuaciones de flujo (2), y nuevamente reemplazando las ecuaciones de flujo en las ecuaciones de nivel (1), obtenemos el sistema de ecuaciones diferenciales ordinarias no lineal (4), que denominaremos el modelo del sistema estrés-enfermedad.

$$
\begin{gathered}
\frac{d}{d t} \text { Estrés }=a \cdot \text { Estrés } \cdot \text { Enfermedad }-b \cdot \text { Estrés } \\
\frac{d}{d t} \text { Enfermedad }=c \cdot \text { Estrés }-d \cdot \text { Enfermedad }
\end{gathered}
$$

Haciendo un conveniente cambio en la notación, donde $x$ es el estrés y la enfermedad esta representada por $y$, obtenemos el modelo matemático presentado en la Ecuación (5) que utilizaremos en el resto del documento. 


$$
\begin{aligned}
& \frac{d x}{d t}=a x y-b x \\
& \frac{d y}{d t}=c x-d y
\end{aligned}
$$

Aunque existen métodos cuantitativos y cualitativos para la medición del estrés [7] y cada variedad de enfermedad, desde el punto de vista de lo explicativo de la relación estrés-enfermedad que se presenta en este artículo, el propósito no es que el modelo pueda ser utilizado en el tratamiento clínico de pacientes, sino que permita la comprensión por escenarios de las posibilidades prospectivas de la relación, orientándose, de este modo, hacia la comprensión sistémica de sus consecuencias, de manera que, pueda ser utilizado como instrumento pedagógico. En ese orden de ideas, en este artículo no se realizarán consideraciones sobre las unidades de medida, ni sobre la promoción de métodos para la medición con los que pudiera ser alimentado el modelo.

\subsection{Análisis de estabilidad del modelo}

Acerca de los puntos de equilibrio del sistema (5) y de su estabilidad, debemos reiterar que los valores de los parámetros $a, b, c, d$ del sistema, son estrictamente mayores que cero. Los puntos de equilibrio calculados son el origen $(0,0)$ y el punto $\left(\frac{b d}{a c}, \frac{b}{a}\right)$. La representación linealizada del sistema es $\dot{X}=J(X) \cdot X$, donde $X=(x, y)^{T} \mathrm{y}$

$$
J(X)=\left(\begin{array}{cc}
a y-b & a x \\
c & -d
\end{array}\right)
$$

es la matriz jacobiana del sistema.

Al evaluar el equilibrio del origen en la jacobiana de la Ecuación (6) obtenemos la matriz

$$
J(0,0)=\left(\begin{array}{cc}
-b & 0 \\
c & -d
\end{array}\right)
$$

cuyos autovalores son $\lambda_{1}=-b$ y $\lambda_{2}=-d$, por lo que decimos que el equilibrio en el origen es un nodo estable.

Cuando evaluamos el equilibrio del punto $\left(\frac{b d}{a c}, \frac{b}{a}\right)$ en la jacobiana de la Ecuación (6) obtenemos la matriz

$$
J\left(\frac{b}{a}, \frac{b c}{a d}\right)=\left(\begin{array}{cc}
0 & \frac{b^{2} d}{a c} \\
c & -d
\end{array}\right)
$$

cuyos autovalores son

$$
\lambda_{1}=-\frac{d}{2}-\frac{1}{2} \sqrt{d^{2}+\frac{b^{2} d}{a}}
$$

$\mathrm{y}$

$$
\lambda_{2}=-\frac{d}{2}+\frac{1}{2} \sqrt{d^{2}+\frac{b^{2} d}{a}}
$$

Es fácil ver que el discriminante es positivo, por lo que no esperamos raíces imaginarias, y se puede demostrar también fácilmente que $\lambda_{1}<0$ y $\lambda_{2}>0$, con lo que el equilibrio no trivial es inestable tipo silla.

Los valores de estabilidad de los puntos de equilibrio, representados en el retrato de fases de la Figura (2(a)), se coinciden con la dinámica esperada en el sistema, como podemos ver si revisamos la cuenca de atracción en la región $R_{1}$ y la cuenca de repulsión en la región $R_{2}$, de la Figura (2(b)). Valores de enfermedad distintos con un bajo nivel de estrés generador del mismo estado de enfermedad, conducen a una desaparición futura de ambos estados. Pero niveles altos de estrés, solo realimentan su relación con la enfermedad provocando estados elevados futuros de estrés y de enfermedad.

\section{Sistemas no suaves}

Muchos procesos físicos son modelados como sistemas dinámicos suaves a tramos (PWS por sus siglas en ingles). Es decir, su movimiento se caracteriza por periodos de evolución suave que son interrumpidos por eventos instantáneos. El análisis tradicional de sistemas dinámicos se ha enfocado en problemas suaves donde estos eventos instantáneos no ocurren, dejando a un lado aquellos sistemas que son no-suaves, como es el caso de la fricción, colisión, sistemas con restricciones intermitentes y procesos con componentes conmutados.

En general los PWS son conjuntos de ecuaciones diferenciales ordinarias (EDOs) en $\mathbb{R}^{n}$, donde el 


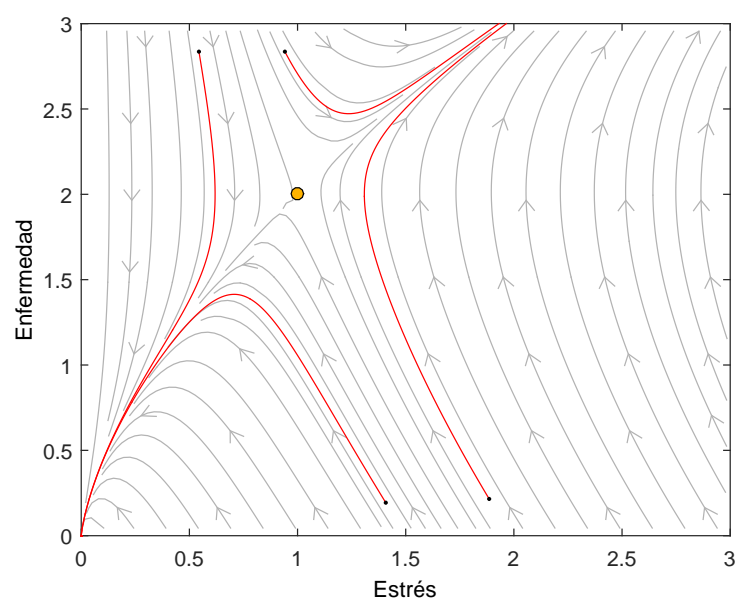

(a)

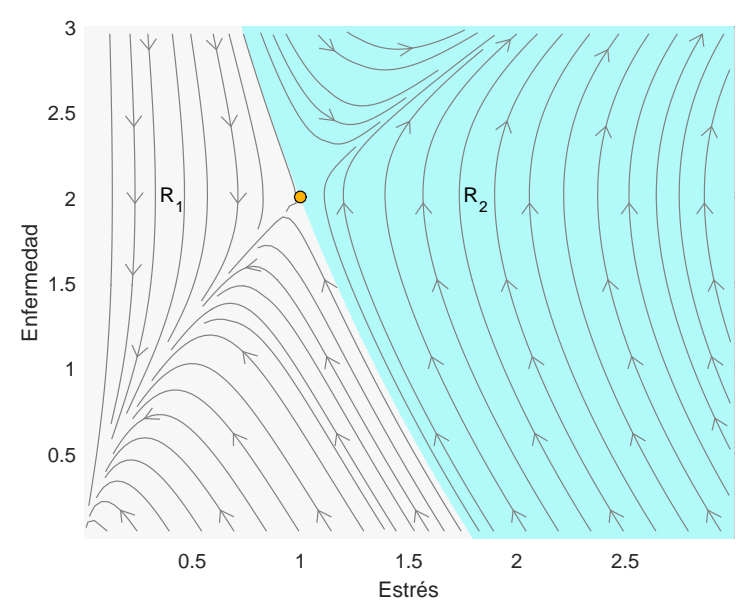

(b)

Figura 2. Retrato de fases y cuencas de atracción del sistema de ecuaciones diferenciales (5).

plano de fases esta dividido en un numero finito de regiones $S_{i}$, tales que $S_{i} \cap S_{j}=\emptyset$, en cada una de las cuales actúa un sistema de EDO's suave $F_{i}$, es decir,

$$
\dot{x}=f(x, \mu) \quad x \in \mathbb{R}^{n}, \quad \mu \in \mathbb{R}^{p},
$$

donde

$$
f(x, \mu)=F_{i}(x, \mu) \quad \forall x \in S_{i} \subset \mathbb{R}^{n}, \quad i=1, \ldots, n,
$$

Cada pareja de regiones $S_{i}$ y $S_{j}$ están separadas por una frontera

$$
\Sigma_{i j}=\left\{x \in \mathbb{R}^{n} \mid h(x)_{i j}=k_{i j}\right\},
$$

denominada región de conmutación, donde $h$ es un campo escalar suave y $k_{i j} \in \mathbb{R}$, de modo que la región de conmutación es de dimensión $(n-1)$. Cuando existe intersección de dos regiones de conmutación $\Sigma_{i j}$, el flujo de la intersección ocurre en una variedad de dimensión $(n-3)$.

De acuerdo a lo anterior, la evolución dinámica de los PWS depende del flujo en cada una de las regiones $S_{i}$ y $\Sigma_{i j}$.

En la literatura se encuentran reportados tres clases de PWS:

1. Los sistemas PWS continuos, donde para cada punto de $\Sigma_{i j}$ se tiene un único vector tangente, es decir, los campos vectoriales de las regiones $S_{i}$ y $S_{j}$ se coinciden en la región de conmutación, haciendo que los flujos crucen suavemente la región de conmutación $\Sigma_{i j}$.

2. Los sistemas de Filippov, que son aquellos en los que para cada punto de $\Sigma_{i j}$ no se tiene necesariamente un único vector tangente, provocando discontinuidad del campo vectorial $f$ y un nuevo campo vectorial resultante $g_{i j}$ en la frontera $\Sigma_{i j}$, que genera lo que se conoce como deslizamientos.

3. Los sistemas con impacto, donde la región $S_{j}$ es una región prohibida del campo vectorial por lo que $\Sigma_{i j}$ se convierte en una región de impacto y el flujo en la región $S_{i}$ es suave.

Este trabajo se centra en el estudio de sistemas de Filippov, por lo tanto no incluye detalles acerca de sistemas PWS continuos y sistemas con impactos. Estos detalles pueden ser consultados en [16, 17].

Dado que en los Sistemas de Filippov los vectores tangentes de cada punto en la región de conmutación $\Sigma_{i j}$ podrían ser no únicos, es necesario realizar dos consideraciones: 1) los tipos de regiones de conmutación que podríamos encontrar y 2) el campo vectorial que actúa en la región de conmutación.

Para la clasificación de las regiones de conmutación ${ }^{5}$, supongamos por simplicidad dos regiones en el plano, que resultan de dividir un conjunto abierto $U$ en la vecindad del cero, por causa de una región de conmutación $\Sigma_{i j}$, como sigue:

\footnotetext{
${ }^{5}$ Guardia M., Seara T.S. and Teixeira M.A. (2011). Generic bifurcations of low codimension of planar Filippov Systems. Journal of Differential Equations. Pág. 1967-2023.
} 


$$
\begin{aligned}
& \Sigma^{+}=\left\{(x, y) \in U \subset \mathbb{R}^{2} \mid h(x, y)>0\right\} \\
& \Sigma^{-}=\left\{(x, y) \in U \subset \mathbb{R}^{2} \mid h(x, y)<0\right\}
\end{aligned}
$$

Denominemos $F_{i}$ al campo vectorial definido en $\Sigma^{+}$y $F_{j}$ al campo vectorial definido en $\Sigma^{-}$. Tenemos entonces la siguiente clasificación de las regiones de conmutación

- Región de cruce: $\Sigma^{c}=\left\{p \in \Sigma i j:\left(F_{i}(p)\right.\right.$. $\left.\nabla h(p)) \cdot\left(F_{j}(p) \cdot \nabla h(p)\right)>0\right\}$

- Región de deslizamiento: $\Sigma^{s}=\{p \in \Sigma i j$ : $\left.F_{i}(p) \cdot \nabla h(p)<0, F_{j}(p) \cdot \nabla h(p)>0\right\}$

- Región de escape: $\Sigma^{e}=\left\{p \in \Sigma i j: F_{i}(p)\right.$. $\nabla h(p)>0,\left(F_{j}(p) \cdot \nabla h(p)<0\right\}$

donde $\nabla h(x)_{i j}$ es el vector gradiente del campo escalar $h(x)_{i j}$ en la región de conmutación $\Sigma_{i j}$. Para definir el campo vectorial que actúa en la región de conmutación se usa el método convexo de Filippov [12], el cual define un único vector tangente sobre cada uno de los puntos de la región de conmutación $\Sigma_{i j} \subset \mathbb{R}^{n}$, a partir de una combinación de los campos vectoriales de las regiones $S_{i}$ y $S_{j}$, generando el campo vectorial $g_{i j}$, como se ve en la Ecuación (11).

$$
g_{i j}=(1-\alpha) F_{i}+\alpha F_{j}, \quad 0 \leq \alpha \leq 1,
$$

donde

$$
\alpha=\frac{\nabla h(x)_{i j} \cdot F_{i}}{\nabla h(x)_{i j} \cdot\left(F_{i}-F_{j}\right)}
$$

Los límites a derecha $\partial \Sigma_{i j}^{+}$e izquierda $\partial \Sigma_{i j}^{-}$(puntos tangente) de la región de deslizamiento $\Sigma_{i j}$ se obtienen cuando $\alpha=1$ y $\alpha=0$, es decir,

$$
\begin{gathered}
\partial \Sigma_{i j}^{+}:=\left\{x \in \Sigma_{i j}: \alpha=1\right\} \\
\partial \Sigma_{i j}^{-}:=\{x \in \Sigma: \alpha=0\}
\end{gathered}
$$

Por otro lado, ya que $g_{i j}$ es un campo vectorial, es posible encontrar puntos en la región de conmutación que satisfacen $g_{i j}=0$, los cuales son denominados pseudo-equilibrios $x_{p} \in \mathbb{R}^{n}$ del campo vectorial $g_{i j}$.
Finalmente, como se trata del caso en que existen fronteras que se cruzan, es necesario detectar el cruce y las ecuaciones de flujo en la intersección $g_{i j k l}$, lo cual depende de los cuatro campos vectoriales involucrados en el cruce, de acuerdo a la ecuación:

$$
g_{i j k l}=(1-\alpha)(1-\beta) F_{i}+(1-\alpha) \beta F_{j}+\alpha(1-\beta) F_{k}+\alpha \beta F_{l}
$$

donde $\alpha$ se define como antes, en la Ecuación (14) $\mathrm{y}$

$$
\beta=\frac{\nabla h(x)_{k l} \cdot F_{k}}{\nabla h(x)_{k l} \cdot\left(F_{k}-F_{l}\right)}
$$

$\operatorname{con} \beta \in[0,1]$. Las constantes $\alpha$ y $\beta$ se encuentran resolviendo simultáneamente el sistema de ecuaciones $\nabla h(x)_{i j} \cdot g_{i j k l}=0$ y $\nabla h(x)_{k l} \cdot g_{i j k l}=0$.

La figura (3(a)) muestra para $\mathbb{R}^{2}$, la intersección de dos regiones de conmutación que por simplicidad se han tomado de manera que coinciden con los ejes coordenados. Esta definición de las regiones de conmutación en el plano, particiona el espacio de estados en las regiones $S_{i}, i=1, \ldots, 4$, y las regiones $\Sigma_{12}, \Sigma_{23}, \Sigma_{34}, \Sigma_{14}$ y $\Sigma_{1234}$. En la Figura (3(b)) se representan los vectores gradiente $\nabla h(x)_{12}=\nabla h(x)_{34}=$ $\nabla h(x)_{a}$ y $\nabla h(x)_{14}=\nabla h(x)_{23}=\nabla h(x)_{b}$ de los campos escalares que definen las regiones de conmutación como sigue:

$$
\begin{aligned}
& \Sigma_{a}=\left\{(x, y) \in \mathbb{R}^{2} \mid x=0\right\} \\
& \Sigma_{b}=\left\{(x, y) \in \mathbb{R}^{2} \mid y=0\right\}
\end{aligned}
$$

El procedimiento utilizado para solucionar numéricamente sistemas de Filippov está basado en la detección de eventos, es decir, determinar el tiempo y las coordenadas donde la evolución del sistema cruza una frontera $\Sigma$ o encuentra un punto tangente sobre la región de conmutación, para de este modo, realizar el cambio al siguiente sistema de EDOs que se debe integrar.

\section{Formulación del sistema no suave}

En la Sección (2) se propuso un sistema de ecuaciones diferenciales para representar la relación estrés-enfermedad, vea Ecuación (5). En esta sección partiremos de la idea de que el espacio de fases 


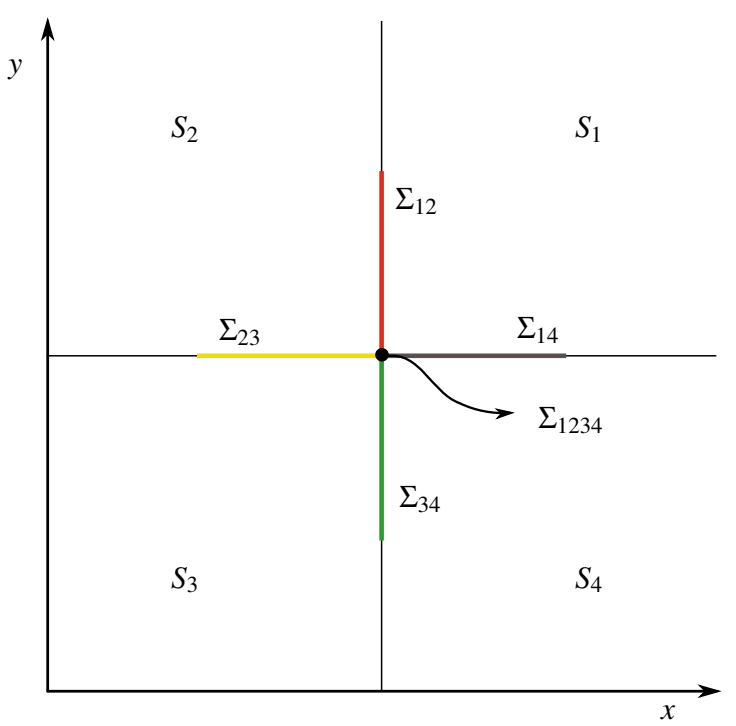

(a)

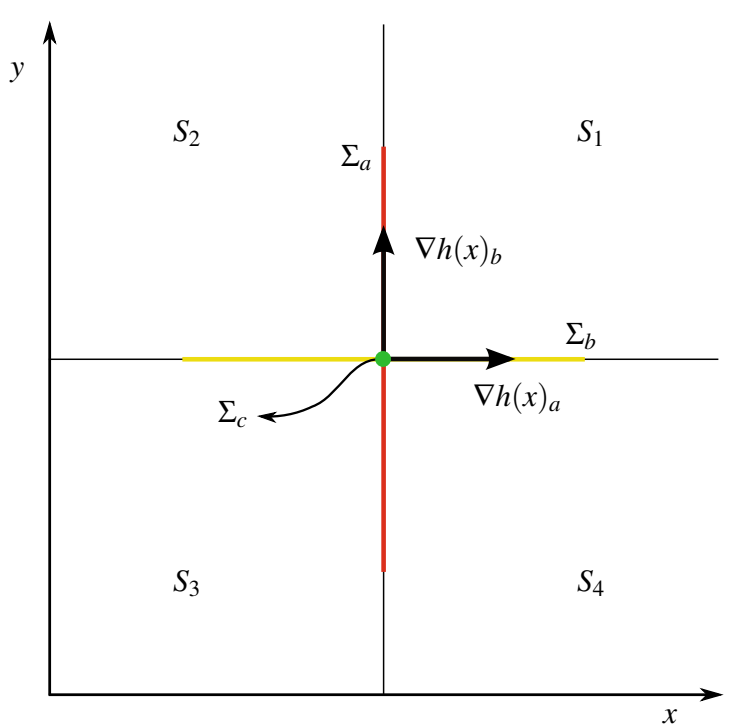

(b)

Figura 3. Intersección de las fronteras de conmutación para el caso plano: (a) Definición de las regiones de conmutación y (b) vectores gradiente de los campos escalares que definen las regiones de conmutación.

debe ser particionado, configurando un Sistema de Filippov, debido a que tanto la enfermedad como el estrés no tienen cambios necesariamente continuos en todo el espacio de estados, sino que, tanto el estrés como la enfermedad, evolucionan por etapas.

Para esta nueva formulación, se consideran valores arbitrarios $0<x_{i}<x_{f}$ del estrés y $0<y_{i}<y_{f}$ de la enfermedad, que se definen empíricamente y son distintos para cada individuo, de modo que, al ser alcanzados el sistema experimente conmutación del campo vectorial, cruzando a otra región del sistema o produciendo deslizamiento.

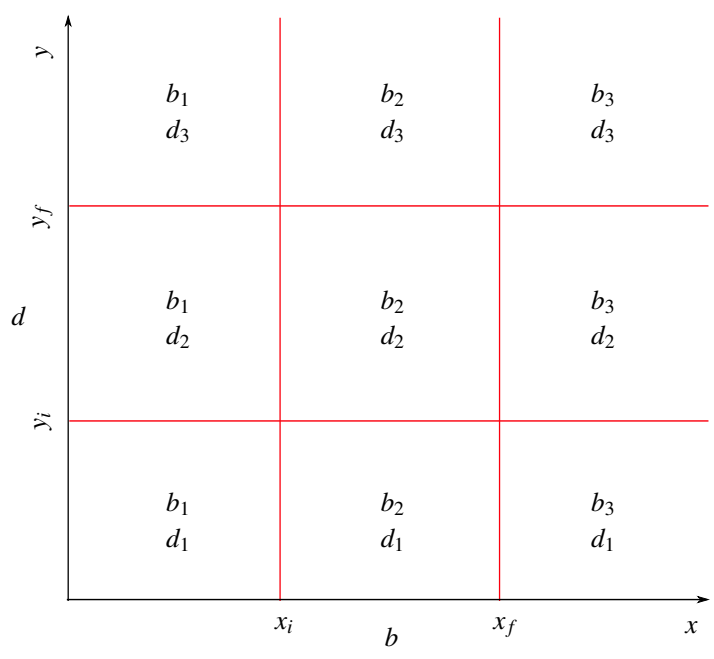

(a)

Figura 4. Partición del primer cuadrante del espacio de estados del sistema donde se han considerado los valores arbitrarios $x_{i}, x_{f}$ de estrés y $y_{i}, y_{f}$ de enfermedad, pero también se han asignado los diferentes valores de los parámetros de frecuencia con la que se recurre a las pseudosoluciones $b$ y frecuencia con la que se sigue un tratamiento médico $d$.

Es decir, se ha particionado el primer cuadrante del espacio de estados del sistema estrés-enfermedad, que es el que en la práctica tiene sentido de ser considerado, y para hacerlo, se consideraron los valores arbitrarios $x_{i}, x_{f}$ de estrés y $y, y_{f}$ de enfermedad, vea Figura (4(a)).

Esta formulación es adecuada para el sistema debido a que tanto el estrés, como la enfermedad, están condicionados por los comportamientos no suaves de los individuos, quienes cambian "por saltos" la frecuencia con la cual recurren a pseudosoluciones para aliviar el estrés o asisten a tratamientos médicos para atender sus enfermedades. Por esta razón, para la nueva formulación se conserva el sistema planar de Ecuaciones (5) sobre cada una de las nuevas regiones del espacio de estados, pero se realizará en cada una de las nuevas regiones, cambios en los valores de los parámetros $b$ y $d$, que corresponden a la frecuencia en la que se recurre a las pseudosoluciones para aliviar el estrés y a la frecuencia en la que se sigue un tratamiento médico a partir de la enfermedad, respectivamente. 
El modelo resultante es como sigue:

$$
\left\{\begin{array}{l}
\dot{x}=a x y-b x \\
\dot{y}=c x-d y
\end{array}\right.
$$

sujeto a las restricciones

$$
\begin{aligned}
& b=\left\{\begin{array}{ccc}
b_{1} & \text { si } & x<x_{i} \\
b_{2} & \text { si } & x_{i}<x<x_{f} \\
b_{3} & \text { si } & x>x_{f}
\end{array}\right. \\
& d=\left\{\begin{array}{ccc}
d_{1} & \text { si } & y<y_{i} \\
d_{2} & \text { si } & y_{i}<y<y_{f} \\
d_{3} & \text { si } & y>y_{f}
\end{array}\right.
\end{aligned}
$$

Donde $b_{1}<b_{2}<b_{3}$ y $d_{1}<d_{2}<d_{3}$, vea Figura (4(a)). El sistema resultante es un Sistema de Filippov.

\section{Resultados}

En la Figura 5 se presentan los resultados de tres de las simulaciones realizadas, mostrando las cuencas de atracción o de repulsión, según sea el caso, de cada uno de los puntos de equilibrio y de pseudoequilibrio del sistema.

En esta discusión de los resultados se ha tenido en cuenta el hecho de que los puntos de equilibrio son asimptóticamente estables y que, por lo tanto, se alcanzan los valores de equilibrio cuando $t \longrightarrow \infty$.

La primera simulación, vea Figura (5(a)), es para el caso en el que existe eventualmente cambios abruptos de la frecuencia con la que se sigue un tratamiento médico, pero no se tienen cambios en la frecuencia con la que se recurre a pseudosoluciones para aliviar el estrés.

Siguiendo la Figura (5(a)), vemos en $R_{1}$ la cuenca de atracción del origen, es decir, el conjunto de todos los estados de un individuo con ciertos niveles de estrés y de enfermedad, que con el tiempo, tenderán a cero, describiendo así la recuperación completa del individuo del cuadro estrés-enfermedad. Por otro lado, en $R_{2}$, tenemos el conjunto de todos los estados que para valores grandes del tiempo, conducen a valores muy grandes de estrés y de enfermedad. Vemos también dos tramos en azul, en las que se genera deslizamiento, denotadas $\Sigma_{S} s$, que corresponderán a aquellos estados en los que el paciente presenta indecisión a someterse de una u otra manera al tratamiento sugerido por el experto médico.

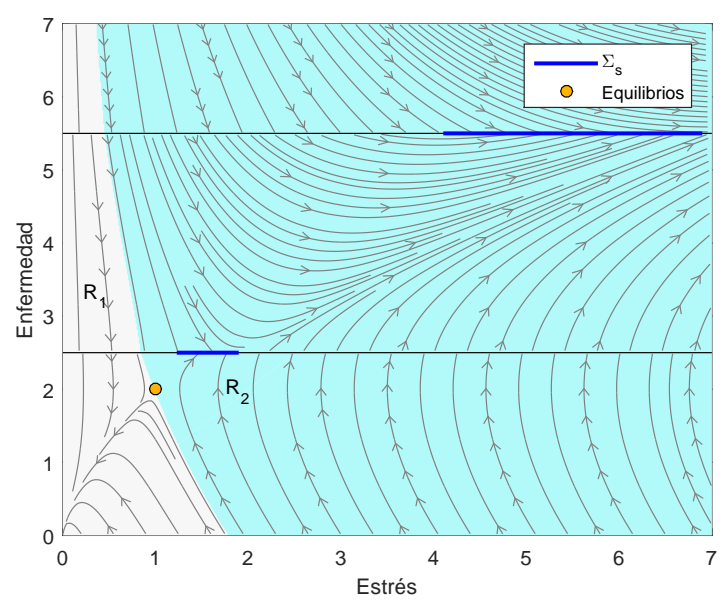

(a)

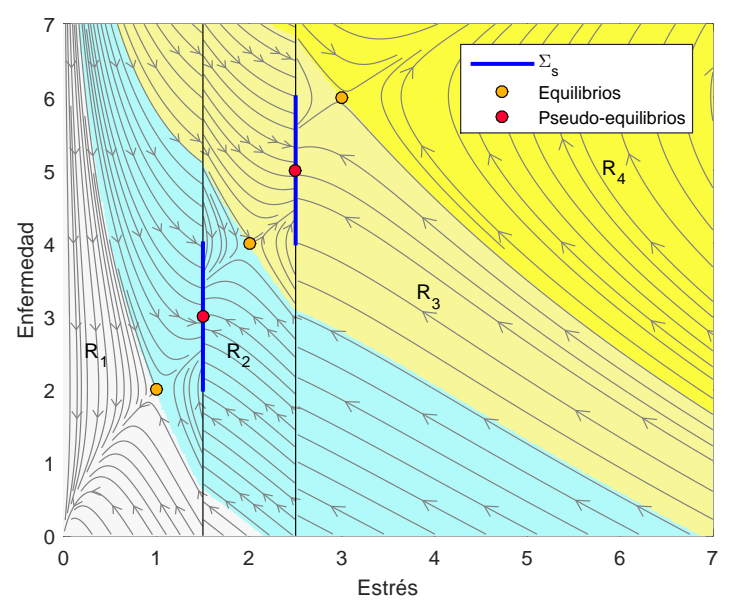

(b)

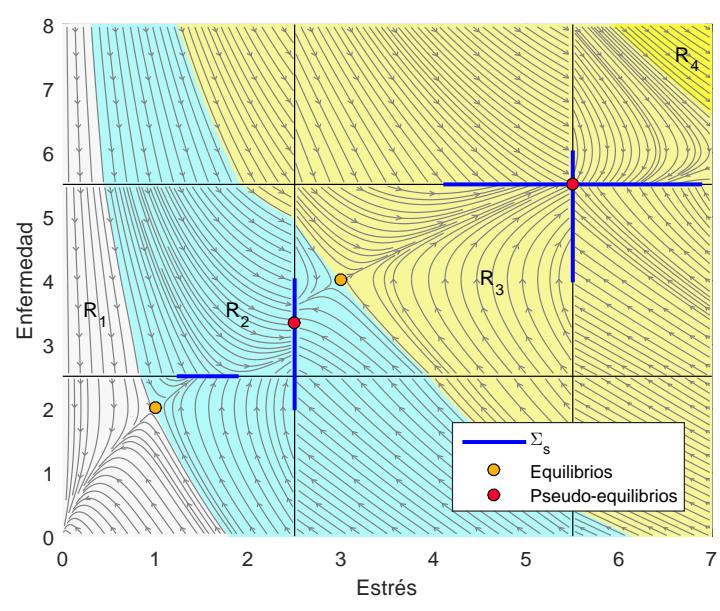

(c)

Figura 5. Retratos de fase del sistema de Filippov de la dupla estrés-enfermedad: (a) regiones de conmutación perpendiculares a $y$, (b) regiones de conmutación perpendiculares a $x$ y (c) regiones de conmutación que se cortan entre sí. 
Los valores utilizados en la simulación de la Figura (5(a)), configuran dos escenarios tendenciales del sistema, uno deseable en el que el paciente puede mejorar completamente, y otro indeseable en el que el paciente solo puede empeorar.

En la Figura (5(b)), se presentan los resultados de la segunda simulación. Ahora tenemos el caso en el que la frecuencia con la que se recurre a pseudosoluciones para aliviar el estrés, eventualmente sufre cambios abruptos, mientras que la frecuencia con la que se sigue un tratamiento médico es fija.

De acuerdo a la Figura (5(b)), ahora tenemos una dinámica mucho más rica, en la que encontramos las cuencas de atracción del origen y de los dos pseudoequilibrios del sistema. Con respecto a las cuencas de atracción de los pseudoequilibrios, los resultados bien podrían referirse a los conjuntos de personas que al experimentar estrés y síntomas de enfermedad recurren a algún tipo de pseudosolución que, si bien no les resuelve el problema, si les permite acercarse a un valor de equilibrio en el que, por la costumbre, se sienten como si estuvieran sanos, manteniendo al margen los estresores y viviendo normalmente con ello. Es interesante que sean los dos pseudoequilibrios los valores estables de estados que previamente se deslizaron por la región de conmutación, ofreciéndonos la idea de que estos estados estables en los que se experimenta el cuadro de estrés-enfermedad, se sostienen por la utilización de más de una pseudosolución.

La Figura (5(b)), nos brinda la imagen de cuatro escenarios dinámicos, en los que se tiene: 1) un escenario deseado en el que el paciente con el tiempo no presentará ni estrés, ni enfermedad (cuenca estable del origen $R_{1}$ ), 2) dos escenarios en los que se termina conviviendo con estrés y enfermedad (cuencas estables de los pseudoequilibrios en $R_{2}$ y $R_{3}$ ) y 3 ) un escenario totalmente indeseable que con el tiempo puede terminar cobrando la vida de la persona $R_{4}$.

Finalmente, para la simulación presentada en la Figura (5(c)), tenemos el caso en el que la frecuencia con la que se recurre a pseudosoluciones para aliviar el estrés y la frecuencia con la que se sigue un tratamiento médico, pueden eventualmente sufrir cambios abruptos. Esta situación es más realista de lo que experimenta un paciente con respecto a las mencionadas frecuencias, porque no es fácil en la práctica librarse de los estresores, bien sea porque el problema generador del estrés toma tiempo en resolverse, es muy costoso o es muy engorroso, cualquiera fuere el caso, el individuo en su necesidad, deberá dar cambios abruptos y cambiar de pseudosoluciones y/o tratamientos médicos.

Siguiendo la Figura (5(c)), encontramos de nuevo la cuenca estable del origen para estados moderados de estrés y enfermedad $R_{1}$. También encontramos cuencas de atracción de los dos pseudoequilibrios del sistema, pero ahora, para el pseudoequilibrio en $R_{2}$, vemos una región de deslizamiento horizontal, dándonos la idea de estabilidad por la dualidad en la implementación de distintas pseudosoluciones, mientras que el pseudoequilibrio en $R_{3}$ demanda además la implementación de distintos tratamientos médicos, junto con la implementación de pseudosoluciones.

De esta manera, tenemos de nuevo cuatro escenarios: 1) un escenario deseado en el que el paciente puede mejorar completamente con el tiempo, 2) un escenario que encuentra un valor de estabilidad por la implementación de diferentes pseudosoluciones, 3) un escenario que se hace estable para valores grandes de estrés y de enfermedad implementando múltiples pseudosoluciones y tratamientos médicos y 4) el escenario indeseado en el que con el tiempo el paciente puede perder la vida.

\section{Conclusiones}

Se construye un modelo matemático de la relación estrés-enfermedad utilizando como metodología de modelamiento la dinámica de sistemas. El modelo es un sistema de ecuaciones diferenciales de primer orden no lineales con un equilibrio estable en el origen y otro equilibrio inestable tipo silla en el primer cuadrante.

Partiendo del modelo suave de la relación estrésenfermedad, se configuró un sistema de Filippov, a partir del cual se puede considerar cambios abruptos eventuales en la frecuencia con la que se recurre a pseudosoluciones para aliviar el estrés y la frecuencia con la que se sigue un tratamiento médico para aliviar la enfermedad generada por el estrés.

Se implementó un algoritmo basado en la detección de eventos para simular sistemas de Filippov con fronteras que se cruzan y se aplicó al modelo planar que describe el proceso dinámico entre el estrés y la enfermedad permitiendo obtener un retrato 
de fases completo que incluye zonas de deslizamiento, puntos tangente, puntos de equilibrio, y pseudoequilibrios bajo valores fijos de los parámetros. Se utilizaron diferentes condiciones iniciales para determinar las diferentes cuencas de atracción en un tiempo de computo relativamente bajo pues este depende fuertemente de la precisión en la detección de los eventos y del tamaño de paso del integrador.

Los resultados son aceptables, con respecto a lo que se espera de la dinámica del comportamiento del sistema representado.

\section{Referencias}

[1] B. B. Berle, M. A. Pinsky, S. W. Wolf y H. G. Wolf, "A Clinical Guide to Prognosis in stress diseases", JAMA, vol. 149 , no. 18, pp. 16241628, 1952.

[2] H. Selye, Stress in Health and Disease,Butterworth, 1976.

[3] B. Cannon, "Walter Bradford Cannon: Reflections on the man and his contributions", International Journal of Stress Management, vol. 1, no. 2, pp. 145-158, 1994.

[4] J. W. Mason, "A re-evaluation of the concept of "non-specificityïn stress theory", Journal of Psychiatric Research, vol. 8, no. 3, pp. 323-333, 1971.

[5] J. P. Henry, "Psychological Factors and Coronary Heart Disease", Holistic Medicine, vol. 2, no. 7, pp. 119-132, 1987.

[6] R. H. Rahe, "Psychosocial stressors and adjustment disorder: van Gogh's life chart illustrates stress and disease.", Journal of Clinical Psychiatry., vol. 51, no. 11, pp. 13-19, 1990.

[7] T. H. Holmes, R. H. Rahe, "The Social Readjustment Rating Scale.”, Journal of Psychosomatic Research, vol. 11, no. 2, pp. 203-210, 1967.
[8] J. Aracil y F. Gordillo, "Dinámica de sistemas", Alianza Editorial Madrid, 1997.

[9] J. D. Sterman, "Business dynamics : systems thinking and modeling for a complex world", McGraw-Hill, 2000.

[10] P. M. Senge, "La quinta disciplina", Granica, 1992.

[11] C. A. Rojas, "Procesos Complejos del Estrés: Dinámica no-lineal", Tesis de Maestría, Universidad Nacional de Colombia, 2012.

[12] A. F. Filippov, "Differential Equations with Discontinuous Righthand Sides", Kluwer Academic Publishers, 1988.

[13] D. Giaouris16, S. Maity, S. Banerjee, V. Pickert, y B. Zahawi, "Application of Filippov Method for the Analysis of Subharmonic Instability in dc-dc Converters", International Journal of Circuit Theory and Application, vol. 37, no. 8, pp. 899-919, 2008.

[14] F. Dercole, A. Gragnanni y S. Rinaldi, "Bifurcation analysis of piecewise smooth ecological models", Theoretical Population Biology, vol. 72, no. 2, pp. 197-213, 2007.

[15] J. Amador, "Smooth and Filippov Models of Sustainable Development: Bifurcations and Numerical Computations", Differential Equations and Dynamical Systems., vol. 21, no. 1, pp. 173-184, 2013.

[16] G. Osorio, M. di Bernardo, y S. Santini, "Corner-Impact Bifurcations: A Novel Class of Discontinuity-Induced Bifurcations in CamFollower Systems", SIAM Journal on Applied Dynamical Systems., vol. 7, no. 1, pp. 18-38, 2008.

[17] M. di Bernardo, C. Budd, A. R. Champneys, y P. Kowalczyk, "Piecewise-smooth Dynamical Systems: Theory and Applications", Springer, 2008. 
